\title{
Hazardous Postoperative Outcomes of Unexpected COVID-19 Infected Patients: A Call for Global Consideration of Sampling all Asymptomatic Patients Before Surgical Treatment
}

\author{
Chen Nahshon $^{1}$ (1) $\cdot$ Arie Bitterman $^{2} \cdot \operatorname{Riad~Haddad~}^{3} \cdot$ David Hazzan $^{4} \cdot$ Ofer Lavie $^{1}$
}

Published online: 16 May 2020

(C) Société Internationale de Chirurgie 2020

\begin{abstract}
Background In December 2019, a novel coronavirus was identified as the cause of many pneumonia cases in China and eventually declared as a pandemic as the virus spread globally. Few reports were published on the outcome of surgical procedures in diagnosed COVID-19 patients and even fewer on the surgical outcomes of asymptomatic undiagnosed COVID-19 surgical patients. We aimed to review all published data regarding surgical outcomes of preoperatively asymptomatic untested coronavirus disease 2019 (COVID-19) patients.

Methods This report is a review on the perioperative period in COVID-19 patients who were preoperatively asymptomatic and not tested for COVID-19. Searches were conducted in PubMed April 4th, 2020. All publications, of any design, were considered for inclusion.

Results Four reports were identified through our literature search, comprising 64 COVID-19 carriers, of them 51 were diagnosed only in the postoperative period. Synthesis of these reports, concerning the postoperative outcomes of patients diagnosed with COVID-19 during the perioperative period, suggested a 14/51 (27.5\%) postoperative mortality rate and severe mostly pulmonic complications, as well as medical staff exposure and transmission.

Conclusions COVID-19 may have potential hazardous implications on the perioperative course. Our review presents results of unacceptable mortality rate and a high rate of severe complications. These observations warrant further well-designed studies, yet we believe it is time for a global consideration of sampling all asymptomatic patients before surgical treatment.
\end{abstract}

Chen Nahshon

csarshalom@gmail.com

1 Department of Gynecologic Surgery and Oncology, Carmel Medical Center, 7 Michal Street, Haifa 3436212, Israel

2 Department of General Surgery A, Carmel Medical Center, Haifa, Israel

3 Department of General Surgery B, Carmel Medical Center, Haifa, Israel

4 Department of General Surgery C, Sheba Medical Center, Tel Hashomer, Sackler School of Medicine, Tel Aviv University, Israel

\section{Introduction}

In December 2019, a novel coronavirus was identified as the cause of many pneumonia cases in Wuhan, China [1]. The new virus, now known as severe acute respiratory syndrome coronavirus 2 (SARS-CoV-2), led to the outbreak of coronavirus disease 2019 (COVID-19), first in China and then worldwide, causing an international emergency. Despite isolation efforts, due to high transmission rates, eventually a pandemic was declared [2,3].

COVID-19 usually presents with fever, cough, dyspnea, myalgia and/or fatigue and may infect any person at any age $[4,5]$. However, many carriers do not present any symptoms $[5,6]$. Thus, differentiating the new virus from 
other known infections based on the clinical presentation is practically impossible. A severe disease, usually presenting in the elderly and in those with an underlying disease, may result in progressive respiratory failure requiring intensive care unit (ICU) hospitalization [5]. Hypertension, cardiovascular disease, diabetes mellitus, smoking, chronic obstructive pulmonary disease (COPD), chronic kidney disease and malignancy were shown to be prevalent underlying diseases among hospitalized COVID-19 patients $[7,8]$. The mortality rate was estimated to be between two and five percent [9].

During the current pandemic, in addition to guidance on staffing, infection control and prevention, guidelines recommended postponing all elective surgeries and procedures [10-12].

Even with elective/non-urgent procedures postponed, urgent and emergent surgeries are still being performed. Operating on COVID-19 patients raises few critical medical issues, including the optimal anesthesiology, surgical approach and the patient's postoperative outcomes, as well as the exposure of medical staff to the in-hospital COVID19 patients. As suggested by different medical unions, all surgical candidate patients must complete preoperative universal health screening for known symptoms of COVID-19.

In addition, special consideration is necessary for evaluating the appropriateness of procedural interventions, recognizing the significant resources they require (i.e., equipment, space and staff).

According to American guidelines, including the Society of Gynecologic Oncology (SGO) and the Society of American Gastrointestinal and Endoscopic Surgeons (SAGES), preoperative patients should be tested for COVID-19, regardless of their symptoms or exposure $[11,13]$.

The question of whether to test all asymptomatic surgical candidate patients of unknown COVID-19 status bothers many surgical staff members and medical authorities. The value of testing asymptomatic surgical candidates of unknown COVID-19 differs between countries and continents and depends on underlying prevalence rates, false negative rates of tests, operation types, timing of the test relative to surgery and various epidemiology principles.

The purpose of this document is to highlight the published outcomes of asymptomatic patients who were operated during the COVID-19 outbreak and were finally found to be infected with the COVID-19 during the postoperative period.

\section{Methods}

This report is a review, proposed to find all relevant data on the perioperative period in COVID-19 patients who were preoperatively asymptomatic and not tested for COVID-19.

Searches were conducted in PubMed April 4th, 2020 (and reconducted on April 10th, 2020) to identify studies reporting the perioperative course of preoperatively unknown asymptomatic COVID-19 patients.

The following search terms were used: COVID-19 or novel coronavirus or SARS-CoV-2 or $2019 \mathrm{nCoV}$ and surgery or surgical or operation or perioperative or postoperative or anesthesia.

All publications of all designs, including original studies, observational studies, case reports, case series and randomized controlled studies were considered for inclusion.

There were no date or language restrictions on the search. References of relevant papers were searched manually for relevant studies.

Due to time constraints, a study protocol was not registered, and one reviewer (C.N) conducted the search, reviewed full texts and extracted data.

\section{Results}

Overall, 430 titles and abstracts were identified through database searching. After screening, 32 potentially relevant reports were evaluated as full text for inclusion. Finally, only four reports were found to be published on perioperative risks during the COVID-19 outbreak. These publications comprise 64 COVID-19 carriers, of them 51 were diagnosed only in the postoperative period [14-17]. Table 1 summarizes the characteristics and outcomes of the undiagnosed COVID-19 surgical patients.

The earliest report describes the perioperative period of three surgical patients (cholecystectomy, hernia repair and hysterectomy) in Tehran, Iran, in February 2020 [14]. The COVID-19 status was unknown for all patients before surgery. All patients developed postoperative fever and pulmonary complications after uneventful operations. Two of the three patients had COVID-19 confirmed by a positive real-time polymerase chain reaction (RT-PCR) test. Of these two COVID-19 postoperatively confirmed patients, one died due to acute respiratory failure and multiple organ failure (MOF). The remaining patient was clinically diagnosed and died due to the postoperative progressive course of COVID-19 infection. An additional patient suffered from acute respiratory distress syndrome (ARDS) one day before his scheduled bariatric surgery, and rapidly 
Table 1 Characteristics and outcomes of the undiagnosed COVID-19 surgical patients

\begin{tabular}{|c|c|c|c|c|c|c|c|}
\hline References & Country & $\begin{array}{l}\text { Number of } \\
\text { patients }\end{array}$ & $\begin{array}{l}\text { Average } \\
\text { age }\end{array}$ & Type of surgery & $\begin{array}{l}\text { Perioperative } \\
\text { complications }\end{array}$ & $\begin{array}{l}\text { Postoperative } \\
\text { fatality }\end{array}$ & Comments \\
\hline $\begin{array}{l}\text { Aminian A } \\
\text { et al. [14] }\end{array}$ & Iran & 3 & 70 & $\begin{array}{l}\text { Cholecystectomy, } \\
\text { Hernia Repair, } \\
\text { Hysterectomy }\end{array}$ & $\begin{array}{l}\text { Fever } \\
\text { Pulmonary } \\
\text { complications (ARDS) } \\
\text { Diarrhea } \\
\text { Acute cardiac injury } \\
\text { Multiple organ failure }\end{array}$ & $\begin{array}{l}2 / 3 \\
66.6 \%\end{array}$ & $\begin{array}{l}\text { An additional } \\
\text { patient died } \\
\text { one day before } \\
\text { planned } \\
\text { surgery }\end{array}$ \\
\hline Qin et al. [15] & China & 1 & 37 & Liver transplantation & $\begin{array}{l}\text { Fever } \\
\text { Pulmonary } \\
\text { complications }\end{array}$ & $\begin{array}{l}0 / 1 \\
0 \%\end{array}$ & $\begin{array}{l}\text { Fever first elevated } \\
\text { after } \\
\text { hepatic arterial } \\
\text { chemoembolization }\end{array}$ \\
\hline Li et al. [16] & China & 13 & 60.2 & $\begin{array}{l}\text { Thoracic Surgery (lung } \\
\text { or esophagus) }\end{array}$ & $\begin{array}{l}\text { Fever } \\
\text { Pulmonary } \\
\text { complications } \\
\text { Diarrhea } \\
\text { Muscular soreness }\end{array}$ & $\begin{array}{l}5 / 13 \\
38.5 \%\end{array}$ & $\begin{array}{l}\text { Severe disease in } 7 \\
\text { patients }\end{array}$ \\
\hline Lei et al. [17] & China & 34 & 55 & All elective surgeries & $\begin{array}{l}\text { Fever } \\
\text { Pulmonary } \\
\text { complications (ARDS) } \\
\text { Myalgia, Arthralgia } \\
\text { Dizziness, Headache, } \\
\text { Nausea, Diarrhea } \\
\text { Secondary infection } \\
\text { Acute cardiac injury } \\
\text { Acute kidney injury }\end{array}$ & $\begin{array}{l}7 / 34 \\
20.6 \%\end{array}$ & $\begin{array}{l}44.1 \% \text { intensive } \\
\text { care unit } \\
\text { admission rate }\end{array}$ \\
\hline
\end{tabular}

Acute Respiratory Distress Syndrome (ARDS)

progressed to cardiopulmonary arrest. PCR was not performed due to rapid deterioration and insufficient testing capacity.

The second publication is a case report detailing the perioperative presentation of COVID-19 in a liver transplant recipient [15]. This 37-year old showed no respiratory abnormalities upon admission. On the following day of hepatic arterial chemoembolization, his fever spiked up to $39{ }^{\circ} \mathrm{C}$. As chemoembolization-associated sterile inflammation was suspected, cephalosporin antibiotic was initiated. In the following days, liver transplant surgery was performed, and immunosuppressive drugs were prescribed. Due to persistent fever and a suspected chest computed tomography (CT), RT-PCR was obtained and confirmed positive for COVID-19. COVID-19 was finally negative on postoperative day (POD) 49, and the patient was discharged from the hospital two months after his surgery.

The third publication entails 25 cases of COVID-19 infection from a single thoracic surgery department [16]. Of these cases, 13 were of hospitalized patients and 12 were of health care staff. Of these 13 patients, seven had a severe disease. 12 patients had a positive nucleic test for COVID-19, while the remaining patient was clinically diagnosed. Reviewing their data, a potential COVID-19 perioperative carrier was identified. This patient had localized ground glass on CT imaging, was misdiagnosed before surgery, and his fever elevated only on POD1. On POD 3, his CT scan already revealed severe COVID-19 infection. On POD 5, the patient died. During his hospital stay, six health care staff members and five postoperational patients were exposed and found to have a positive nucleic test for COVID-19. As mortality rate was significantly high in postoperative patients $(5 / 13)$, the authors show that chest surgery was significantly associated with death due to COVID-19. Moreover, they demonstrate that age and COPD were significantly associated with a severe presentation and finally death from COVID-19.

The fourth report details the postoperative outcomes of 34 asymptomatic patients who had underwent elective surgery during the COVID-19 outbreak in Wuhan, China [17]. Of the 34 patients, 20 had one or more comorbidities, most commonly hypertension, malignancy, diabetes and 
cardiovascular disease. The surgeries were classified by technical difficulty, general complexity and the level of risk. Most surgeries were defined to be of mild (level-2) or moderate (level-3) risks. All patients suffered postoperatively from COVID-19 pneumonia, commonly complicated by ARDS, shock, secondary infection, cardiological complications and acute kidney injury. 15 patients (44.1\%) required ICU admission due to MOF or mechanical ventilation. Most of these patients underwent level-3 surgeries, while non-ICU patients were mostly after surgeries of milder risk and shorter duration. In addition, ICU patients were significantly older (55 years vs. 47 years) and more probable to have an underlying comorbidity. Seven patients (20.6\%) eventually died due to progressive respiratory failure, all during the postoperative period of level-3 surgeries, all with at least one underlying comorbidity. As patients developed COVID-19 symptoms after an average of 2.6 days, the authors deduce that the elective surgery was performed during the asymptomatic incubation period of COVID-19 and that surgery may accelerate and exacerbate COVID-19 disease.

\section{Discussion}

Small case series are reporting on the outcome of surgical procedures in patients diagnosed with COVID-19. Moreover, there are limited case series reporting on the impact of COVID-19 in undiagnosed preoperative patients. Our review brings to light part of the surgical practice challenges during the COVID-19 outbreak, and specifically aims to describe the hazardous postoperative outcomes of undiagnosed COVID-19 patients, emphasizing the importance of considering preoperative COVID-19 screening.

The rationale behind knowing the COVID-19 status of any surgical candidate is ensuring patient safety, reduction of postoperative complications [18] and potential transmission of the novel coronavirus from an unknown carrier to medical staff or to other patients [19]. While spreading the virus in the operation room and during hospital stay is potentially managed by personal protective equipment [20], the complications that may present during patients' recovery are not under our control.

The recent accumulation of published data concerning the postoperative outcome of patients diagnosed with COVID-19 during the perioperative period suggested a $14 / 51(27.5 \%)$ postoperative mortality rate and severe mostly pulmonic complications. The latest report by Lei $\mathrm{S}$ et al. [17] suggested $20 \%$ postoperative death, $44 \%$ ICU admission rate and $100 \%$ postoperative pneumonia among 34 asymptomatic COVID-19 patients who underwent elective surgery.
Noticeable are the age differences between studies and their association with fatality rate. In line with previously published studies, and as seen in Table 1, the studies with an older average age resulted in higher fatality rates. Though the data were retrieved from a small available number of publications, this morbidity and mortality risk is unacceptable and could be minimized or even avoided primarily by the preoperative diagnosis of patients' COVID-19 status, as suggested by the American guidelines.

With that said, like all screening tests, a true benefit from preoperative screening depends on COVID-19 local prevalence rates and the availability of an accurate objective test. Screening is of extra importance in situations that may lead to different clinical management approaches for the patients and staff.

To elaborate on these key issues, RT-PCR COVID-19 testing was shown to have high specificity with moderate sensitivity, resulting in cases of false negative results [21]. Usually, these false results are a consequence of incorrect sampling [22] and could be addressed by education of health care staff. Adequate sampling, good laboratory standards and high-quality RT-PCR kits, all reduce inaccurate results [22].

Today and especially in the near future, when isolation and quarantine measures ease up, and expecting the comeback of elective surgeries, knowing the preoperative COVID-19 status will be of great clinical importance. The preoperative diagnosis will lead health care providers to use the right protocols aiming to reduce postoperative complications and fatal morbidity. Most importantly, a known COVID-19 status will improve the medical staff decision making concerning the use of protective equipment aiming to prevent the hazardous staff infection. A preoperative screening test may also assist in the decision whether to delay a scheduled operation, and eventually ease answering the risk-benefit question of surgery versus COVID-19 exposure and complications.

The COVID-19 pandemic has presented unique challenges and learning opportunities for surgical centers. The future trajectory of the COVID-19 pandemic is uncertain and during this worldwide pandemic the situation is dynamic, policies and recommendations may change at any time. But since our global overarching goal is to continue providing compassionate, safe care for surgical patients and for medical staff, we believe that preoperative screening for COVID-19 baseline status and detection of asymptomatic surgical candidates during the COVID-19 incubation period is an acceptable requirement. Consideration of preoperative COVID-19 testing may facilitate the preoperative risk and benefit assessment whether to perform or postpone the individual's surgical procedure during the current pandemic. 
In conclusion, COVID-19 may have potential serious implications on the perioperative course and may result in substantial fatality rates. The presented rates were accumulated from a small number of studies and patients, therefore, future well-designed studies are warranted. Nevertheless, knowing the preoperative COVID-19 status before any surgical treatment should be considered especially in high prevalence areas. We believe that in light of the observed outcomes, and in order to protect patients and health care staff, a consideration of sampling asymptomatic patients before surgical treatment is needed.

Funding The authors did not receive funding for this work.

\section{Compliance with ethical standards}

Conflict of interest The authors declare no conflict of interest.

\section{References}

1. Tan W, Zhao X, Ma X et al (2020) A novel coronavirus genome identified in a cluster of pneumonia cases-Wuhan, China 2019-2020. China CDC Wkly 2:61-62

2. Li Q, Guan X, Wu P et al (2020) Early transmission dynamics in Wuhan, China, of Novel Coronavirus-Infected Pneumonia. N Engl J Med. https://doi.org/10.1056/nejmoa2001316

3. World Health Organization (2020) COVID-19 strategic preparedness and response plan: operational planning guidelines to support country preparedness and response, pp 1-20

4. Young BE, Ong SWX, Kalimuddin S et al (2020) Epidemiologic features and clinical course of patients infected with SARS-CoV2 in Singapore. JAMA J Am Med Assoc. https://doi.org/10.1001/ jama.2020.3204

5. Greenland JR, Michelow MD, Wang L, London MJ (2020) COVID-19 infection: implications for perioperative and critical care physicians. Anesthesiology. https://doi.org/10.1097/ALN. 0000000000003303

6. Zou L, Ruan F, Huang M et al (2020) SARS-CoV-2 viral load in upper respiratory specimens of infected patients. N Engl J Med. https://doi.org/10.1056/NEJMc2001737

7. Yang J, Zheng Y, Gou X et al (2020) Prevalence of comorbidities in the novel Wuhan coronavirus (COVID-19) infection: a systematic review and meta-analysis. Int J Infect Dis. https://doi.org/ 10.1016/j.ijid.2020.03.017

8. Emami A, Javanmardi F, Pirbonyeh N, Akbari A (2020) Prevalence of underlying diseases in hospitalized patients with
COVID-19: a systematic review and meta-analysis. Arch Acad Emerg Med 8:e35

9. Wu Y-C, Chen C-S, Chan Y-J (2020) The outbreak of COVID19: An overview. J Chin Med Assoc 83:217-220. https://doi.org/ 10.1097/JCMA.0000000000000270

10. ACS COVID-19: Recommendations for Management of Elective Surgical Procedures. Publ March 13, 2020 facs.org/covid19/clinical-guidance/elective-case/cancer-surgery

11. Dowdy S, Fader AN Surgical Considerations for Gynecologic Oncologists During the COVID-19 Pandemic. 1-4

12. ESSO Statement on COVID-19 Advice for surgical oncologists on cancer service. Publ March 27, 2020 https//www.essoweb.org/ news/esso-statement-covid-19/

13. Pryor A sages and EAES recommendations regarding surgical response TO Rationing of Services: procedural Considerations: Publ March 29, 2020 https//www.sages.org/recommendationssurgical-response-covid-19/

14. Aminian A, Safari S, Razeghian-Jahromi A et al (2020) COVID19 outbreak and surgical practice: unexpected fatality in perioperative period. Ann Surg. https://doi.org/10.1097/SLA. 0000000000003925

15. Qin J, Wang H, Qin X et al (2020) Perioperative presentation of COVID-19 disease in a liver transplant recipient. Hepatology. https://doi.org/10.1002/hep.31257

16. Li YK, Peng S, Li LQ et al (2020) Clinical and transmission characteristics of Covid-19-a retrospective study of 25 cases from a single thoracic surgery department. Curr Med Sci 40:1-6. https://doi.org/10.1007/s11596-020-2176-2

17. Lei S, Jiang F, Su W et al (2020) Clinical characteristics and outcomes of patients undergoing surgeries during the incubation period of COVID-19 infection. EClinicalMedicine. https://doi. org/10.1016/j.eclinm.2020.100331

18. Sabiston textbook of surgery: the biological basis of modern surgical practice. Seventeenth edition/Courtney M. Townsend Jr. ... [and others]. Philadelphia, Elsevier Saunders (2016)

19. Spinelli A, Pellino G (2020) COVID-19 pandemic: perspectives on an unfolding crisis. Br J Surg. https://doi.org/10.1002/bjs. 11627

20. Dexter F, Parra MC, Brown JR, Loftus RW (2020) Perioperative COVID-19 defense: an evidence-based approach for optimization of infection control and operating room management. Anesth Analg. https://doi.org/10.1213/ANE.0000000000004829

21. Zitek T (2020) The appropriate use of testing for COVID-19. West J Emerg Med. https://doi.org/10.5811/westjem.2020.4. 47370

22. Tahamtan A, Ardebili A (2020) Real-time RT-PCR in COVID-19 detection: issues affecting the results. Expert Rev. Mol, Diagn

Publisher's Note Springer Nature remains neutral with regard to jurisdictional claims in published maps and institutional affiliations. 